\title{
On Quasi-Polarized Manifolds Whose Sectional Genus is Equal to the Irregularity
}

\author{
YosHIAKI FUKUMA
}

ABstRACT - Let $(X, L)$ be a quasi-polarized manifold of dimension $n$. In our previous paper, we proved that if $\operatorname{dim} X=3$ and $h^{0}(L) \geq 2$, then $g(X, L) \geq h^{1}\left(\mathcal{O}_{X}\right)$ holds. Here $g(X, L)$ denotes the sectional genus of $(X, L)$. In this paper, we give the classification of quasi-polarized 3-folds $(X, L)$ with $h^{0}(L) \geq 3$ and $g(X, L)=h^{1}\left(\mathcal{O}_{X}\right)$. Moreover as an application of this result, we also give the classification of polarized manifolds $(X, L)$ with $\operatorname{dim} \operatorname{Bs}|L|=1, h^{0}(L) \geq n$ and $g(X, L)=h^{1}\left(\mathcal{O}_{X}\right)$.

\section{Introduction.}

Let $(X, L)$ be a quasi-polarized manifold with $\operatorname{dim} X=n$. For this pair $(X, L)$, the sectional genus $g(X, L)$ is defined by the following formula:

$$
g(X, L)=1+\frac{1}{2}\left(K_{X}+(n-1) L\right) L^{n-1},
$$

where $K_{X}$ is the canonical bundle of $X$. Then there is the following conjecture which was proposed by Fujita [7, (13.7) Remark].

CONJECTURe 1.1 (Fujita). Let $(X, L)$ be a quasi-polarized manifold. Then $g(X, L) \geq q(X)$, where $q(X):=\operatorname{dim} H^{1}\left(\mathcal{O}_{X}\right)$ is the irregularity of $X$.

For this conjecture, there are some results (see [9], [10], [12] and so on). But it is unknown whether this conjecture is true or not even for the case of $\operatorname{dim} X=2$. If $\operatorname{dim} X=2$, then this conjecture is true if $h^{0}(L)>0$ (see [9]). Moreover the classification of quasi-polarized surfaces $(X, L)$ with $g(X, L)=q(X)$ and $h^{0}(L) \geq 1$ was obtained (see [8], [9]).

If $\operatorname{dim} X=3$ and $h^{0}(L) \geq 2$, it is known that this conjecture is true, and

Indirizzo dell'A.: Department of Mathematics, Faculty of Science, Kochi University, Akebono-cho, Kochi 780-8520, Japan

E-mail: fukuma@kochi-u.ac.jp 
the classification of polarized 3-folds $(X, L)$ with $g(X, L)=q(X)$ and $h^{0}(L) \geq 3$ was given (see [12]).

In this paper, we will give the classification of quasi-polarized 3-folds with $g(X, L)=q(X)$ and $h^{0}(L) \geq 3$. As an application of this result, we are able to give the classification of polarized $n$-fold $(X, L)$ with $g(X, L)=q(X)$, $\operatorname{dim} \operatorname{Bs}|L|=1$ and $h^{0}(L) \geq n$. (Here we note that $g(X, L) \geq q(X)$ holds if $\operatorname{dim} \mathrm{Bs}|L|=1$.)

The author would like to thank the referee for giving some useful comments.

\section{Preliminaries.}

Definition 2.1. Let $X$ and $Y$ be projective varieties with $\operatorname{dim} X>\operatorname{dim} Y \geq 1$, and let $f: X \rightarrow Y$ be a surjective morphism with connected fibers. Then $(f, X, Y)$ is called a fiber space. Moreover if $L$ is a nef and big (resp. an ample) line bundle on $X$, then $(f, X, Y, L)$ is called a quasipolarized (resp. polarized) fiber space.

Lemma 2.1. Let $X$ and $C$ be smooth projective varieties with $\operatorname{dim} X=n$ and $\operatorname{dim} C=1$, and let $L$ be a nef and big line bundle on $X$. Assume that there exists a fiber space $f: X \rightarrow C$ such that $h^{0}\left(K_{F}+L_{F}\right) \neq 0$ for a general fiber $F$ off. Then $f_{*}\left(K_{X / C}+L\right)$ is ample.

Proof. First we note that there exists a natural number $m$ such that $(m L)^{n}-n(m L)^{n-1} F>0$. Then by [3, Lemma 4.1], there exists a natural number $k$ such that $\mathcal{O}_{X}(k(m L-F))$ has a nontrivial global section. Hence we have an injective map $\mathcal{O}_{X}(k F) \rightarrow \mathcal{O}(k m L)$. On the other hand, there exists a line bundle $\mathcal{N}$ on $C$ such that $\mathcal{O}(k F)=f^{*}(\mathcal{N})$. Hence by [4, Corollary $1.9]$ we see that $f_{*}\left(K_{X / C}+L\right)$ is ample and we get the assertion.

Definition 2.2. (i) Let $\left(X_{1}, L_{1}\right)$ and $\left(X_{2}, L_{2}\right)$ be quasi-polarized varieties. Then $\left(X_{1}, L_{1}\right)$ and $\left(X_{2}, L_{2}\right)$ are said to be birationally equivalent if there is another variety $G$ with birational morphisms $g_{i}: G \rightarrow X_{i}(i=1,2)$ such that $g_{1}^{*} L_{1}=g_{2}^{*} L_{2}$.

(ii) Let $\left(f_{1}, X_{1}, Y, L_{1}\right)$ and $\left(f_{2}, X_{2}, Y, L_{2}\right)$ be quasi-polarized fiber spaces. Then $\left(f_{1}, X_{1}, Y, L_{1}\right)$ and $\left(f_{2}, X_{2}, Y, L_{2}\right)$ are said to be birationally equivalent if there is another variety $G$ with birational morphisms $g_{i}: G \rightarrow X_{i}$ $(i=1,2)$ such that $g_{1}^{*} L_{1}=g_{2}^{*} L_{2}$ and $f_{1} \circ g_{1}=f_{2} \circ g_{2}$. 
DEFINITION 2.3. Let $X$ be a normal projective variety of dimension $n$ and let $D$ be a $Q$-divisor on $X$. Then $D$ is said to be generically nef if $D L_{1} \cdots L_{n-1} \geq 0$ for any collection of ample Cartier divisors $L_{1}, \ldots, L_{n-1}$ on $X$.

DeFinition 2.4. Let $(X, L)$ be a quasi-polarized variety of dimension $n$. Then the 4 -genus $\Delta(X, L)$ of $(X, L)$ is defined by the following:

$$
\Delta(X, L)=n+L^{n}-h^{0}(L) .
$$

Proposition 2.1. Let $(X, L)$ be a quasi-polarized manifold of dimension $n$. If $K_{X}+(n-1) L$ is not generically nef, then $\Delta(X, L)=0$ or $(X, L)$ is birationally equivalent to a scroll over a smooth curve.

Proof. See [16, Proposition 1.3].

\section{Main results.}

First we will prove the following theorem.

THeOREm 3.1. Let $(f, X, C, L)$ be a quasi-polarized fiber space such that $X$ and $C$ are smooth with $\operatorname{dim} X=n$ and $\operatorname{dim} C=1$. Then $g(X, L) \geq$ $\geq g(C)$. Moreover if $g(X, L)=g(C)$, then $(X, L)$ is one of the following two types.

(a) $\Delta(X, L)=0$.

(b) The pair $(X, L)$ is birationally equivalent to a scroll over $C$.

Proof. (1) If $g(C)=0$, then $g(X, L) \geq 0=g(C)$ by [16, Theorem 1.1]. Moreover if $g(X, L)=0=g(C)$, then by [16, Theorem 1.2] we have $\Delta(X, L)=0$.

(2) Next we assume that $g(C) \geq 1$.

(2.1) First we assume that $K_{X}+(n-1) L$ is generically nef. Then by $[15,1.2$ Theorem] we see that there exists a natural number $j$ with $1 \leq j \leq n-1$ such that $h^{0}\left(K_{X}+j L\right)>0$. Hence $h^{0}\left(K_{F}+j L_{F}\right)>0$ for any general fiber $F$ of $f$. Then $f_{*}\left(K_{X / C}+j L\right) \neq 0$. By Lemma 2.1 we see that $f_{*}\left(K_{X / C}+j L\right)$ is ample. By the same argument as [10, Lemma 1.4.1], we get $\left(K_{X / C}+j L\right) L^{n-1}>0$. Since $1 \leq j \leq n-1$, we have $\left(K_{X / C}+\right.$ $+(n-1) L) L^{n-1}>0$. Then

$$
\begin{aligned}
g(X, L) & =g(C)+\frac{1}{2}\left(K_{X / C}+(n-1) L\right) L^{n-1}+(g(C)-1)\left(\left(L_{F}\right)^{n-1}-1\right) \\
& >g(C) .
\end{aligned}
$$


(2.2) Next we assume that $K_{X}+(n-1) L$ is not generically nef. Then by Proposition 2.1 we see that $\Delta(X, L)=0$ or there exist a quasi-polarized variety $\left(X^{\prime}, L^{\prime}\right)$, a smooth projective variety $M$ and birational morphisms $\mu_{1}: M \rightarrow X$ and $\mu_{2}: M \rightarrow X^{\prime}$ such that $\left(X^{\prime}, L^{\prime}\right)$ is a scroll over a smooth curve.

If $\Delta(X, L)=0$, then we infer that $h^{1}\left(\mathcal{O}_{X^{\prime}}\right)=h^{1}\left(\mathcal{O}_{X}\right)=0$ (see [12, Lemma 1.15]). Hence $g(C)=0$ and this contradicts the assumption of $g(C)>0$. So we may assume that $\left(X^{\prime}, L^{\prime}\right)$ is a scroll over a smooth curve $B$. Let $f^{\prime}: X^{\prime} \rightarrow B$ be its fibration and let $h:=f^{\prime} \circ \mu_{2}: M \rightarrow B$. Then for any general fiber $F_{h}$ of $h$, we have $h^{1}\left(\mathcal{O}_{F_{h}}\right)=0$. Since $g(C)>0$, we see that $f \circ \mu_{1}\left(F_{h}\right)$ is a point. Therefore by [2, Lemma 4.1.13] there exists a surjective morphism $\delta: B \rightarrow C$ such that $f \circ \mu_{1}=\delta \circ h$. But since $f$ and $f^{\prime}$ have connected fibers, we see that $\delta$ is an isomorphism. On the other hand, we can easily check that $g\left(X^{\prime}, L^{\prime}\right)=g(B)$. So we get $g(X, L)=g\left(X^{\prime}, L^{\prime}\right)=$ $=g(B)=g(C)$. Therefore we get the assertion.

REMARK 3.1. There exists an example of a quasi-polarized fiber space $(f, X, C, L)$ such that $g(X, L)=g(C)$ and $(X, L)$ is birationally equivalent to $(V, H)$ with $\Delta(V, H)=0$. For example, let $(V, H)=\left(\mathbb{P}^{n}, \mathcal{O}_{\mathrm{p}^{n}}(1)\right)$. Then we can easily see that $\Delta(V, H)=0$. We take two general members $H_{1}$ and $H_{2}$ in $|H|$ and let $\Lambda$ be a pencil which is generated by $H_{1}$ and $H_{2}$. By using this pencil, we can make a fiber space over a smooth curve. Namely, there exist a smooth projective variety $X$, a birational morphism $\mu: X \rightarrow \mathbb{P}^{n}$ and a fiber space $f: X \rightarrow C$ over a smooth curve $C$. We set $L:=\mu^{*}\left(\mathcal{O}_{\mathbb{p}^{n}}(1)\right)$. Since $q(X)=0$, we see that $C \cong \mathbb{P}^{1}$. Moreover $g(X, L)=g\left(\mathbb{P}^{n}, \mathcal{O}_{\mathbb{P}^{n}}(1)\right)=0=g(C)$ and $(X, L)$ is birationally equivalent to $(V, H)$.

Next we consider quasi-polarized manifolds $(X, L)$ with $\operatorname{dim} X=3$, $h^{0}(L) \geq 3$ and $g(X, L)=q(X)$.

Theorem 3.2. Let $(X, L)$ be a quasi-polarized 3-fold. Assume that $h^{0}(L) \geq 3$. If $g(X, L)=q(X)$, then $(X, L)$ satisfies one of the following two types.

(a) $\Delta(X, L)=0$.

(b) The pair $(X, L)$ is birationally equivalent to a scroll over a smooth curve $C$.

Proof. By [6, Theorem 4.2], there exists a quasi-polarized variety $\left(X^{\prime}, L^{\prime}\right)$ which is birationally equivalent to $(X, L)$ and satisfies one of the 
following conditions:

(i) $K_{X^{\prime}}+2 L^{\prime}$ is nef for the canonical Q-bundle $K_{X^{\prime}}$;

(ii) $\Delta(X, L)=\Delta\left(X^{\prime}, L^{\prime}\right)=0$;

(iii) $\left(X^{\prime}, L^{\prime}\right)$ is a scroll over a curve,

where $X^{\prime}$ is a normal projective variety with only Q-factorial terminal singularities. Since $g(X, L)=g\left(X^{\prime}, L^{\prime}\right)$ and $q(X)=q\left(X^{\prime}\right)$, we may assume that $X$ has only Q-factorial terminal singularities and $(X, L)$ satisfies one of the above conditions.

If $(X, L)$ is the type (ii), then $g(X, L)=0$ by [6, (1.7) Corollary] and $q(X)=0$ by [12, Lemma 1.15]. Hence we obtain $g(X, L)=q(X)$ in this case.

If $(X, L)$ is the type (iii), then we can check $g(X, L)=q(X)$ by easy calculation.

So we may assume that $K_{X}+2 L$ is nef. Let $\pi: \widetilde{X} \rightarrow X$ be a resolution of $X$ such that $\widetilde{X} \backslash \pi^{-1}(\operatorname{Sing}(X)) \cong X \backslash \operatorname{Sing}(X)$, and $\widetilde{L}=\pi^{*}(L)$. Then $h^{0}(\widetilde{L})=$ $=h^{0}(L) \geq 3$. Let $\Lambda$ be a linear pencil which is contained in $|\widetilde{L}|$ such that $\Lambda=\Lambda_{M}+Z$, where $\Lambda_{M}$ is the movable part of $\Lambda$ and $Z$ is the fixed part of $|\widetilde{L}|$. We will make a fiber space by using this $\Lambda$. Let $\varphi: \widetilde{X} \rightarrow \mathbb{P}^{1}$ be the rational map associated with $\Lambda_{M}$, and $\theta: \widetilde{X}^{\prime} \rightarrow \widetilde{X}$ an elimination of indeterminacy of $\varphi$. So we obtain a surjective morphism $\varphi^{\prime}: \widetilde{X}^{\prime} \rightarrow \mathbb{P}^{1}$. If necessary, we take the Stein factorization $\delta: C \rightarrow \mathbb{P}^{1}$ of $\varphi^{\prime}$. Then we have a fiber space $f^{\prime}: \widetilde{X}^{\prime} \rightarrow C$ such that $\varphi^{\prime}=\delta \circ f^{\prime}$. Let $F^{\prime}$ be a general fiber of $f^{\prime}$ and let $a:=\operatorname{deg} \delta$. We consider this quasi-polarized fiber space $\left(f^{\prime}, \widetilde{X}^{\prime}, C, \theta^{*}(\widetilde{L})\right)$. By the proof of [12, Theorem 2.1], we see that there exists a quasi-polarized fiber space $\left(f_{1}, X_{1}, C, L_{1}\right)$ which is birationally equivalent to $\left(f^{\prime}, \widetilde{X}^{\prime}, C, \theta^{*}(\widetilde{L})\right)$ such that $\left(f_{1}, X_{1}, C, L_{1}\right)$ satisfies one of the following conditions.

- $K_{X_{1}}+2 L_{1}$ is $f_{1}$-nef.

- $\left(f_{1}, X_{1}, C, L_{1}\right)$ is a scroll.

If $\left(f_{1}, X_{1}, C, L_{1}\right)$ is a scroll, then we see that $g(X, L)=q(X)$ and this is the type (b) in Theorem 3.2. So we may assume that $K_{X_{1}}+2 L_{1}$ is $f_{1}$-nef. In this case, by [14, Lemma 0.2], we see that $K_{X_{1} / C}+2 L_{1}$ is nef.

(a) The case of $g(C) \geq 1$. Then $\theta$ is the identity map. So we have $\widetilde{X}^{\prime}=\widetilde{X}$ and $\theta^{*}(\widetilde{L})=\widetilde{L}$. By the construction of the fiber space $\left(f^{\prime}, \widetilde{X}^{\prime}, C, \theta^{*}(\widetilde{L})\right)$, we get $\widetilde{L}=\sum_{i=1}^{a} F_{i}+Z$, where each $F_{i}$ is a fiber of $f^{\prime}$ and $Z$ is the fixed part of $|\widetilde{L}|$. Then there exists an ample line bundle $P \in \operatorname{Pic}(C)$ such that $\sum_{i=1}^{a} F_{i}=\left(f^{\prime}\right)^{*}(P)$. In particular $\operatorname{deg} P=a$. 
Claim 3.1. $a \geq 3$.

Proof. First we note that $h^{0}(L)=h^{0}(\widetilde{L})=h^{0}\left(\sum_{i=1}^{a} F_{i}+Z\right)=$ $=h^{0}\left(\sum_{i=1}^{a} F_{i}\right)=h^{0}(P)$. Since $h^{0}(L) \geq 3$, we have $h^{0}(P) \geq 3$. If $a \leq 2$, then $\Delta(C, P)=1+\operatorname{deg} P-h^{0}(P)=1+a-h^{0}(P) \leq 0$. On the other hand, since $P$ is an ample line bundle on $C$, we have $\Delta(C, P) \geq 0$ by [5, Corollary 1.10] or [7, (4.2) Theorem]. Therefore $\Delta(C, P)=0$. But then $C \cong \mathbb{P}^{1}$ (see [5, Lemma $3.1]$ ) and this contradicts the assumption that $g(C) \geq 1$. Hence we have $a \geq 3$.

Here we note that $\widetilde{L}$ is numerically equivalent to $a F^{\prime}+Z$ by the construction above. By the same argument as in the proof of [12, Claim 2.2], we have

$$
\left(K_{\widetilde{X} / C}+2 \widetilde{L}\right)(\widetilde{L})^{2} \geq t\left(K_{\widetilde{X} / C}+2 \widetilde{L}\right)(\widetilde{L}) F^{\prime}
$$

for any natural number $t$ with $t \leq a$. Hence $\left(K_{\widetilde{X} / C_{\widetilde{L}}}+2 \widetilde{L}\right)(\widetilde{L})^{2} \geq$ $\geq 3\left(K_{\widetilde{X} / C}+2 \widetilde{L}\right)(\widetilde{L}) F^{\prime}$ holds because $a \geq 3$. Since $g(C) \geq 1$ and $\left(\widetilde{L}_{F^{\prime}}\right)^{2} \geq 1$, we get

$$
\begin{aligned}
g(\widetilde{X}, \widetilde{L}) & =1+\frac{1}{2}\left(K_{\widetilde{X}}+2 \widetilde{L}\right)(\widetilde{L})^{2} \\
& =g(C)+\frac{1}{2}\left(K_{\widetilde{X} / C}+2 \widetilde{L}\right)(\widetilde{L})^{2}+(g(C)-1)\left(\left(\widetilde{L}_{F^{\prime}}\right)^{2}-1\right) \\
& \geq g(C)+\frac{3}{2}\left(K_{\widetilde{X} / C}+2 \widetilde{L}\right)(\widetilde{L}) F^{\prime} \\
& =g(C)+3 g\left(F^{\prime},\left.\widetilde{L}\right|_{F^{\prime}}\right)+\frac{3}{2}(\widetilde{L})^{2} F^{\prime}-3 .
\end{aligned}
$$

Since $h^{0}\left(\left.\widetilde{L}\right|_{F^{\prime}}\right)>0$ and $\operatorname{dim} F^{\prime}=2$ we have $g\left(F^{\prime},\left.\widetilde{L}\right|_{F^{\prime}}\right) \geq q\left(F^{\prime}\right)$ by [9, Lemma $1.2(2)]$. Because $g(C)+q\left(F^{\prime}\right) \geq q(\widetilde{X})$, we have

$$
g(\widetilde{X}, \widetilde{L}) \geq q(\widetilde{X})+2 g\left(F^{\prime},\left.\widetilde{L}\right|_{F^{\prime}}\right)+\frac{3}{2}(\widetilde{L})^{2} F^{\prime}-3 .
$$

Since $g(\widetilde{X}, \widetilde{L})=g(X, L)=q(X)=q(\widetilde{X})$ holds, we get $2 g\left(F^{\prime},\left.\widetilde{L}\right|_{F^{\prime}}\right)+$ $+\frac{3}{2}(\widetilde{L})^{2} F^{\prime}-3 \leq 0$. Hence we have $g\left(F^{\prime},\left.\widetilde{L}\right|_{F^{\prime}}\right)=0$. Therefore $\kappa\left(F^{\prime}\right)=-\infty$ and by $\left[9\right.$, Theorem 2.1] we have $q\left(F^{\prime}\right)=0$. So $q(\widetilde{X})=g(C)$ because $g(C)=q\left(F^{\prime}\right)+g(C) \geq q(\widetilde{X}) \geq g(C)$. Hence we obtain $g(\widetilde{X}, \widetilde{L})=g(C)$, and by Theorem 3.1 we get the assertion in this case.

(b) The case of $g(C)=0$. Let $\gamma:=\pi \circ \theta$.

(b.1) If $a \geq 2$, then 


$$
\begin{aligned}
g(X, L) & =g(\widetilde{X}, \widetilde{L}) \\
& =g\left(\widetilde{X}^{\prime}, \theta^{*}(\widetilde{L})\right) \\
& =1+\frac{1}{2} \gamma^{*}\left(K_{X}+2 L\right)\left(\theta^{*}(\widetilde{L})\right)^{2} \\
& \geq 1+\gamma^{*}\left(K_{X}+2 L\right)\left(\theta^{*}(\widetilde{L})\right) F^{\prime}
\end{aligned}
$$

because $K_{X}+2 L$ is nef and $a \geq 2$. Let $\widetilde{D}:=\theta\left(F^{\prime}\right)$. By [12, Claims 2.3 and 2.4], we have

$$
\begin{aligned}
g(X, L) & \geq 1+\gamma^{*}\left(K_{X}+2 L\right)\left(\theta^{*}(\widetilde{L})\right) F^{\prime} \\
& =1+\theta^{*}\left(\pi^{*}\left(K_{X}\right)+2 \widetilde{L}\right)\left(\theta^{*}(\widetilde{L})\right) F^{\prime} \\
& =1+\theta^{*}\left(K_{\widetilde{X}}+2 \widetilde{L}\right)\left(\theta^{*}(\widetilde{L})\right) F^{\prime} \\
& \geq 1+\left(\theta^{*}\left(K_{\widetilde{X}}+\widetilde{D}\right)+\theta^{*}(\widetilde{L})\right)\left(\theta^{*}(\widetilde{L})\right) F^{\prime} \\
& \geq 1+\left(K_{\widetilde{X^{\prime}}}+F^{\prime}+\theta^{*}(\widetilde{L})\right)\left(\theta^{*}(\widetilde{L})\right) F^{\prime} \\
& =2 g\left(F^{\prime},\left.\theta^{*}(\widetilde{L})\right|_{F^{\prime}}\right)-1 .
\end{aligned}
$$

Since $\operatorname{dim} F^{\prime}=2$ and $h^{0}\left(\left.\theta^{*}(\widetilde{L})\right|_{F^{\prime}}\right)>0$, we have $g\left(F^{\prime},\left.\theta^{*}(\widetilde{L})\right|_{F^{\prime}}\right) \geq q\left(F^{\prime}\right)$ by [9, Lemma $1.2(2)]$. Moreover since $q\left(F^{\prime}\right)=q\left(F^{\prime}\right)+g(C) \geq q\left(\tilde{X}^{\prime}\right)=q(X)$, we get $g(X, L) \geq 2 q(X)-1$. Therefore $q(X) \leq 1$ because $g(X, L)=q(X)$. In particular $g(X, L) \leq 1$. From [6, Corollaries (4.8) and (4.9)], we see that $(X, L)$ is birationally equivalent to one of the types (a) and (b) in Theorem 3.2. (Here we use the assumption that $g(X, L)=q(X)$.)

(b.2) Here we assume that $a=1$. Then $h^{0}\left(\left.\theta^{*}(\widetilde{L})\right|_{F^{\prime}}\right) \geq 2$. By the same argument as in Case (2) in the proof of [12, Theorem 2.1] we have

$$
q(X)=g(X, L) \geq g\left(F^{\prime},\left.\theta^{*}(\widetilde{L})\right|_{F^{\prime}}\right) \geq q\left(F^{\prime}\right) \geq q(X) .
$$

Hence we have $\kappa\left(F^{\prime}\right)=-\infty$ by [9, Theorem 3.1] since $g\left(F^{\prime},\left.\theta^{*}(\widetilde{L})\right|_{F^{\prime}}\right)=q\left(F^{\prime}\right)$ and $h^{0}\left(\left.\theta^{*}(\widetilde{L})\right|_{F^{\prime}}\right) \geq 2$. Moreover we get

$$
q\left(\tilde{X}^{\prime}\right)=q(X)=q\left(F^{\prime}\right) .
$$

Here we apply the relatively minimal model theory for the fibration $f^{\prime}: \widetilde{X}^{\prime} \rightarrow C \cong \mathbb{P}^{1}$. Since $\kappa\left(F^{\prime}\right)=-\infty$, we see that there exist smooth projective varieties $X^{\sharp}$ and $T$ with $\operatorname{dim} X^{\sharp}=3$ and $1=\operatorname{dim} C \leq \operatorname{dim} T \leq 2$, a birational morphism $\delta^{\sharp}: X^{\sharp} \rightarrow \widetilde{X}^{\prime}$ and surjective morphisms $\delta_{1}: X^{\sharp} \rightarrow T$ and $\delta_{2}: T \rightarrow C$ with connected fibers such that $f^{\prime} \circ \delta^{\sharp}=\delta_{2} \circ \delta_{1}$ and $F_{\delta_{1}}$ is birationally equivalent to a Fano manifold, where $F_{\delta_{1}}$ is a general fiber of $\delta_{1}$. In particular $q\left(F_{\delta_{1}}\right)=0$. We put $f^{\sharp}:=f^{\prime} \circ \delta^{\sharp}$. 
(b.2.1) Assume that $\operatorname{dim} T=1$. Then $\delta_{2}$ is an isomorphism. Hence $q(T)=0$ because $C \cong \mathbb{P}^{1}$. On the other hand, since $q\left(F_{\delta_{1}}\right)=0$, we have $q(X)=q\left(X^{\sharp}\right)=q(T)=0$. Thus we get $g(X, L)=0$ from the assumption that $g(X, L)=q(X)$. Therefore by [6, (4.8) Corollary] we get the assertion.

(b.2.2) Next we assume that $\operatorname{dim} T=2$. If $q(X) \leq 1$, then we have $g(X, L) \leq 1$ and by [6, Corollaries (4.8) and (4.9)] we get the assertion. So we may assume that $q(X) \geq 2$. Let $F_{\delta_{2}}$ (resp. $F^{\sharp}$ ) be a general fiber of $\delta_{2}$ (resp. $f^{\sharp}$ ). Then

$$
\left.\delta_{1}\right|_{F^{\sharp}}: F^{\sharp} \rightarrow F_{\delta_{2}}
$$

is a surjective morphism with connected fibers. Since a general fiber of $\left.\delta_{1}\right|_{F^{\sharp}}$ is $\mathbb{P}^{1}$, we have $q\left(F^{\sharp}\right)=q\left(F_{\delta_{2}}\right)$. On the other hand, we have $q\left(X^{\sharp}\right)=q(T)$ because any general fiber of $\delta_{1}$ is $\mathbb{P}^{1}$, and we have $q\left(F^{\sharp}\right)=q\left(X^{\sharp}\right)$ by (1). So we get $q(T)=q\left(F^{\sharp}\right)=q\left(F_{\delta_{2}}\right)=q\left(F_{\delta_{2}}\right)+q\left(\mathbb{P}^{1}\right)$. Now we are assuming that $q\left(X^{\sharp}\right)=q(X) \geq 2$, so we have $q\left(F_{\delta_{2}}\right) \geq 2$. Therefore, considering the fiber space $\delta_{2}: T \rightarrow C \cong \mathbb{P}^{1}$, we see from [1, Lemme] or [9, Lemma 1.5] that $T$ is birationally equivalent to $F_{\delta_{2}} \times \mathbb{P}^{1}$. In particular $\kappa(T)=-\infty$. So, taking the Albanese map of $T$, there exists a morphism $\alpha: T \rightarrow B$, where $B$ is a smooth projective curve with $g(B)=q(T)=q\left(F_{\delta_{2}}\right)$. Then $\alpha \circ \delta_{1}: X^{\sharp} \rightarrow B$ has connected fibers. Moreover since $q\left(X^{\sharp}\right)=q\left(F^{\sharp}\right)=q\left(F_{\delta_{2}}\right)=g(B)$ we obtain $g\left(X^{\sharp},\left(\theta \circ \delta^{\sharp}\right)^{*}(\widetilde{L})\right)=g(X, L)=q(X)=q\left(X^{\sharp}\right)=g(B)$. Since $\left(X^{\sharp},\left(\theta \circ \delta^{\sharp}\right)^{*}(\widetilde{L})\right)$ is a quasi-polarized 3-fold, we get the assertion by Theorem 3.1.

Here we want to propose the following conjecture which is a quasipolarized manifolds' version of [12, Conjecture 2.15].

CONJECTURe 3.1. Let $(X, L)$ be a quasi-polarized $n$-fold. Assume that $h^{0}(L) \geq n$. If $g(X, L)=q(X)$, then $(X, L)$ is one of the following.

(a) $\Delta(X, L)=0$.

(b) The pair $(X, L)$ is birationally equivalent to a scroll over a smooth curve.

REMARK 3.2. If $n=2$ (resp. $n=3$ ), then this conjecture is true by [9, Theorem 3.1] (resp. Theorem 3.2 above).

Let $(X, L)$ be a polarized manifold of dimension $n$. If $\mathrm{Bs}|L|=\emptyset$ (resp. $\operatorname{dim} \mathrm{Bs}|L|=0$ ), then by [2, Theorem 7.2.10] (resp. [11, Theorem 3.2]) we see that $g(X, L) \geq q(X)$. Moreover, we can get a classification of $(X, L)$ with $g(X, L)=q(X)$ and $\operatorname{dim} \operatorname{Bs}|L| \leq 0$ (see [17, (3.6) Theorem] and [11, Theorem 3.2]). So, as the next step, we consider the case where $\operatorname{dim} \operatorname{Bs}|L|=1$. 
THeorem 3.3. Let $(X, L)$ be a polarized manifold of dimension $n \geq 3$. Assume that $\operatorname{dim} \mathrm{Bs}|L|=1$.

(i) The inequality $g(X, L) \geq q(X)$ holds.

(ii) Furthermore we assume that $h^{0}(L) \geq n$. If $g(X, L)=q(X)$, then $(X, L)$ is one of the following.
(a) $\left(\mathbb{P}^{n}, \mathcal{O}_{\mathrm{P}^{n}}(1)\right)$.
(b) $\left(\mathrm{Q}^{n}, \mathcal{O}_{\mathrm{Q}^{n}}(1)\right)$.
(c) A scroll over a smooth curve.

Proof. From the assumption, we see that there exist an $(n-3)$-ladder $X \supset X_{1} \supset \cdots \supset X_{n-3}$ such that each $X_{j}$ is a normal and Gorenstein projective variety of dimension $n-j$ (see [13, Proposition 1.12 (2)]). Let $L_{j}=L_{X_{j}}$ for every $j$ with $1 \leq j \leq n-3$. Then we see that

$$
h^{1}\left(\mathcal{O}_{X}\right)=h^{1}\left(\mathcal{O}_{X_{1}}\right)=\cdots=h^{1}\left(\mathcal{O}_{X_{n-3}}\right)
$$

and

$$
g(X, L)=g\left(X_{1}, L_{1}\right)=\cdots=g\left(X_{n-3}, L_{n-3}\right) .
$$

Let $\pi: M_{n-3} \rightarrow X_{n-3}$ be a resolution of $X_{n-3}$. Then

$$
g\left(M_{n-3}, \pi^{*}\left(L_{n-3}\right)\right)=g\left(X_{n-3}, L_{n-3}\right)
$$

and

$$
h^{1}\left(\mathcal{O}_{M_{n-3}}\right) \geq h^{1}\left(\mathcal{O}_{X_{n-3}}\right) .
$$

(i) Here we note that $h^{0}\left(L_{n-3}\right) \geq 2$. Hence by [12, Theorem 2.1] we have

$$
g\left(M_{n-3}, \pi^{*}\left(L_{n-3}\right)\right) \geq q\left(M_{n-3}\right) .
$$

Therefore by (2), (3), (4), (5) and (6), we get $g(X, L) \geq q(X)$.

(ii) Assume that $h^{0}(L) \geq n$. Then $h^{0}\left(L_{n-3}\right) \geq 3$. If $g(X, L)=q(X)$, then by (2), (3), (4), (5) and (6) we have $g\left(M_{n-3}, \pi^{*}\left(L_{n-3}\right)\right)=q\left(M_{n-3}\right)$ and $q\left(M_{n-3}\right)=$ $=q\left(X_{n-3}\right)$. In particular, $X_{n-3}$ has the Albanese map (see [2, Remark 2.4.2]). Let $\alpha: X_{n-3} \rightarrow \operatorname{Alb}\left(X_{n-3}\right)$ be its Albanese map, where $\operatorname{Alb}\left(X_{n-3}\right)$ is the Albanese variety of $X_{n-3}$. Then $\alpha \circ \pi: M_{n-3} \rightarrow \operatorname{Alb}\left(X_{n-3}\right)$ is the Albanese map of $M_{n-3}$. Since $\left(M_{n-3}, \pi^{*}\left(L_{n-3}\right)\right)$ is a quasi-polarized 3 -fold with $h^{0}\left(\pi^{*}\left(L_{n-3}\right)\right) \geq 3$ and $g\left(M_{n-3}, \pi^{*}\left(L_{n-3}\right)\right)=q\left(M_{n-3}\right)$, we can apply Theorem 3.2. Then $\left(M_{n-3}, \pi^{*}\left(L_{n-3}\right)\right)$ satisfies one of the following types:

- $\Delta\left(M_{n-3}, \pi^{*}\left(L_{n-3}\right)\right)=0$.

- $\left(M_{n-3}, \pi^{*}\left(L_{n-3}\right)\right)$ is birationally equivalent to a scroll over a smooth curve. 
If $\Delta\left(M_{n-3}, \pi^{*}\left(L_{n-3}\right)\right)=0$, then $g(X, L)=g\left(M_{n-3}, \pi^{*}\left(L_{n-3}\right)\right)=0$. Therefore we get the assertion from Fujita's results (see [7, (12.1) Theorem and (5.10) Theorem]).

Next we assume that $\left(M_{n-3}, \pi^{*}\left(L_{n-3}\right)\right)$ is birationally equivalent to a scroll over a smooth curve. Let $(V, H)$ be its scroll. If $h^{1}\left(\mathcal{O}_{V}\right)=0$, then we see that $g(X, L)=0$ and we get the assertion. So we may asssume that $h^{1}\left(\mathcal{O}_{V}\right) \geq 1$. Then the dimension of the image of Albanese map of $V$ is one because $(V, H)$ is a scroll over a smooth curve. Since $M_{n-3}$ and $V$ are birationally equivalent each other, we see that the dimension of the image of $\alpha \circ \pi$ is also one. Hence the dimension of the image of $\alpha$ is also one. Since $h^{1}\left(\mathcal{O}_{V}\right)>0$ implies $h^{1}\left(\mathcal{O}_{X}\right)>0$, we can take the Albanese map $\beta: X \rightarrow \operatorname{Alb}(X)$ of $X$.

Claim 3.2. $\operatorname{dim} \beta(X)=1$.

Proof. First we consider a map $b: X_{n-3} \hookrightarrow X \rightarrow \operatorname{Alb}(X)$. By the universality of the Albanese map, there exists a morphism $c: \operatorname{Alb}\left(X_{n-3}\right) \rightarrow$ $\rightarrow \operatorname{Alb}(X)$ such that $c \circ \alpha=b$. On the other hand, since $\operatorname{dim} \alpha\left(X_{n-3}\right)=1$, we have $\operatorname{dim} b\left(X_{n-3}\right)=\operatorname{dim}(c \circ \alpha)\left(X_{n-3}\right) \leq \operatorname{dim} \alpha\left(X_{n-3}\right)=1$. But by [2, Propositions 5.1.1 and 5.1.2] we have $\operatorname{dim} b\left(X_{n-3}\right) \geq 1$ because $\operatorname{dim} \beta(X) \geq 1$. Hence $\operatorname{dim} b\left(X_{n-3}\right)=1$. Furthermore by using [2, Propositions 5.1.1 and 5.1.2], we also see $\operatorname{dim} \beta(X)=1$.

Since $\operatorname{dim} \beta(X)=1$, we find that $\beta(X)$ is smooth and $\beta: X \rightarrow \beta(X)$ is a fiber space over a smooth curve $\beta(X)$. Let $C=\beta(X)$. Since $h^{1}\left(\mathcal{O}_{X}\right)=g(C)$, we get $g(X, L)=g(C)$. By [10, Theorem 1.4.2] we see that $(X, L)$ is a scroll over $C$. So we get the assertion.

REMark 3.3. (i) Theorem 3.3 shows that [12, Conjecture 2.15] is true for the case of $\operatorname{dim} \operatorname{Bs}|L|=1$.

(ii) If $\operatorname{dim} \operatorname{Bs}|L| \leq 0$, then we see that $h^{0}(L) \geq n$. Hence by [17, (3.6) Theorem] and [11, Theorem 3.2] we infer that [12, Conjecture 2.15] is true for the case of $\operatorname{dim} \operatorname{Bs}|L| \leq 0$.

\section{REFERENCES}

[1] A. Beauville, L'inegalite $p_{g} \geq 2 q-4$ pour les surfaces de type général, Bull. Soc. Math. France, 110 (1982), pp. 343-346.

[2] M. C. BeltrametTi - A. J. Sommese, The adjunction theory of complex projective varieties, de Gruyter Expositions in Math. 16, Walter de Gruyter, Berlin, NewYork (1995). 
[3] J. P. Demaily, Effective bounds for very ample line bundles, Invent. Math., 124 (1996), pp. 243-261.

[4] H. ESNAULT - E. VIEHWEG, Effective bounds for semipositive sheaves and for the height of points on curves over complex function fields, Composit. Math., 76 (1990), pp. 69-85.

[5] T. FujITA, On the structure of polarized varieties with 4-genera zero, J. Fac. Sci. Univ. of Tokyo, 22 (1975), pp. 103-115.

[6] T. FuJITA, Remarks on quasi-polarized varieties, Nagoya Math. J., 115 (1989), pp. 105-123.

[7] T. Fujita, Classification Theories of Polarized Varieties, London Math. Soc. Lecture Note Ser., 155, Cambridge University Press (1990).

[8] Y. Fukuma, On polarized surfaces $(X, L)$ with $h^{0}(L)>0, \kappa(X)=2$, and $g(L)=q(X)$, Trans. Amer. Math. Soc., 348 (1996), pp. 4185-4197.

[9] Y. FuKUMA, A lower bound for the sectional genus of quasi-polarized surfaces, Geom. Dedicata, 64 (1997), pp. 229-251.

[10] Y. Fukuma, A lower bound for sectional genus of quasi-polarized manifolds, J. Math. Soc. Japan, 49 (1997), pp. 339-362.

[11] Y. Fukuma, On the nonemptiness of the linear system of polarized manifolds, Canad. Math. Bull., 41 (1998), pp. 267-278.

[12] Y. Fukuma, On sectional genus of quasi-polarized 3-folds, Trans. Amer. Math. Soc., 351 (1999), pp. 363-377.

[13] Y. FuKUma, On the sectional geometric genus of quasi-polarized varieties, II, Manuscripta Math., 113 (2004), pp. 211-237.

[14] Y. Funuma, A lower bound for sectional genus of quasi-polarized manifolds, II, preprint, http://www.math.kochi-u.ac.jp/fukuma/preprint.html

[15] A. HöRIng, On a conjecture of Beltrametti and Sommese, arXiv:0912.1295, to appear in J. Algebraic Geom.

[16] A. HöRIng, The sectional genus of quasi-polarised varieties, Arch. Math., 95 (2010), pp. 125-133.

[17] A. J. Sommese, On the adjunction theoretic structure of projective varieties, Complex analysis and algebraic geometry (Göttingen, 1985), pp. 175-213, Lecture Notes in Math., 1194 (Springer, Berlin, 1986).

Manoscritto pervenuto in redazione il 20 luglio 2010. 
